Article

\title{
Analysis of CFTR Mutation Spectrum in Ethnic Russian Cystic Fibrosis Patients
}

\author{
Nika V. Petrova '*, Nataliya Y. Kashirskaya (D), Tatyana A. Vasilyeva, Elena I. Kondratyeva (D), \\ Elena K. Zhekaite , Anna Y. Voronkova, Victoria D. Sherman D, Varvara A. Galkina, \\ Eugeny K. Ginter, Sergey I. Kutsev, Andrey V. Marakhonov (D) and Rena A. Zinchenko $\mathbb{D}$ \\ Research Centre for Medical Genetics, Moskvorechje Street, 1, 115478 Moscow, Russia; \\ kashirskayanj@mail.ru (N.Y.K.); vasilyeva_debrie@mail.ru (T.A.V.); elenafpk@mail.ru (E.I.K.); \\ elena_zhekayte@gmail.com (E.K.Z.); voronkova111@yandex.ru (A.Y.V.); tovika@yandex.ru (V.D.S.); \\ vgalka06@rambler.ru (V.A.G.); ekginter@mail.ru (E.K.G.); kutsev@mail.ru (S.I.K.); \\ marakhonov@generesearch.ru (A.V.M.); renazinchenko@mail.ru (R.A.Z.) \\ * Correspondence: npetrova63@mail.ru
}

Received: 30 March 2020; Accepted: 13 May 2020; Published: 15 May 2020

\begin{abstract}
The distribution and frequency of the CFTR gene mutations vary considerably between countries and ethnic groups. Russians are an East Slavic ethnic groups are native to Eastern Europe. Russians, the most numerous people of the Russian Federation (RF), make about $80 \%$ of the population. The aim is to reveal the molecular causes of CF in ethnic Russian patients as comprehensively as possible. The analysis of most common CFTR mutations utilized for CF diagnosis in multiethnic RF population accounts for about $83 \%$ of all CF-causing mutations in 1384 ethnic Russian patients. Variants c.1521_1523delCTT (F508del), c.54-5940_273+10250del21kb (CFTRdele2,3), c.2012delT (2143delT), c.2052_2053insA (2184insA), and c.3691delT (3821delT) are most typical for CF patients of Russian origin. DNA of 154 CF patients, Russian by origin, in whom at least one mutant allele was not previously identified (164 CF alleles), was analyzed by Sanger sequencing followed by the multiplex ligase-dependent probe amplification (MLPA) method. In addition to the 29 variants identified during the previous test for common mutations, 91 pathogenic CFTR variants were also revealed: 29 missense, 19 nonsense, 14 frame shift in/del, 17 splicing, 1 in frame ins, and 11 copy number variations $(\mathrm{CNV})$. Each of the 61 variants was revealed once, and 17 twice. Each of the variants c.1209G >C (E403D), c.2128A > T (K710X), c.3883delA (4015delA), and c.3884_3885insT (4016insT) were detected for three, c.1766+1G >A $(1898+1 G>A)$ and c. $2834 \mathrm{C}>\mathrm{T}$ (S945L) for four, c.1766+1G $>$ C (1898+1G>C) and c.(743+1_744-1)_(1584+1_1585-1)dup (CFTRdup6b-10) for five, c.2353C>T (R785X) and c.4004T $>C$ (L1335P) for six, c.3929G $>$ A (W1310X) for seven, c.580-1G $>$ T (712-1G $>$ T for eight, and c.1240_1244delCAAAA (1365del5) for 11 unrelated patients. A comprehensive analysis of CFTR mutant alleles with sequencing followed by MLPA, allowed not only the identification of 163 of 164 unknown alleles in our patient sample, but also expansion of the mutation spectrum with novel and additional frequent variants for ethnic Russians.
\end{abstract}

Keywords: cystic fibrosis; CFTR gene; common and new pathogenic variants; ethnic Russian population

\section{Introduction}

Cystic fibrosis (CF, OMIM\#219700) is an autosomal recessive condition resulting from the pathogenic variants in the CF transmembrane regulator (CFTR) gene. CF is a hereditary disease caused by impaired epithelial chloride channel CFTR function. Variants are classified as disease causing, not disease causing, of variable clinical significance, or of unknown clinical significance. More than 2000 different variants of the CFTR gene sequence have been revealed, the pathogenicity of $20 \%$ of 
which is established [1,2]. In many populations the most frequent pathogenic variant of the CFTR gene (ABCC7) is F508del, which accounts for approximately two thirds of all CFTR alleles, with a decreasing prevalence from Northwest to Southeast Europe. The remaining third of alleles are substantially heterogeneous, with fewer than 20 mutations occurring at a worldwide frequency of more than $0.1 \%$. Some variants can reach a higher frequency in certain populations, due to a founder effect in religious, ethnic or geographical isolates [3]. The spectrum and frequency of CFTR gene sequence variants vary significantly in different countries and ethnic groups, which suggests the development of regional molecular diagnostics protocols to optimize medical and genetic care for CF patients [4].

The diagnosis of CF was proven by typical pulmonary or gastrointestinal symptoms or positive neonatal screening, or the diagnosis of CF in a sibling, as well as at least one of the following: two positive sweat chloride tests, or the identification of two CFTR pathologic variants in trans according to the guidelines of the European Cystic Fibrosis Society as well as the Russian National Consensus on Cystic Fibrosis [5,6].

Molecular genetic studies on CF have been conducted in the Laboratory of Genetic Epidemiology of the Research Centre for Medical Genetics for a long period of time starting from the year 1989. To date, the laboratory has analyzed the DNA of more than $3400 \mathrm{CF}$ patients, the clinical diagnosis was confirmed in the Scientific-Clinical Department for Cystic Fibrosis of the Research Centre for Medical Genetics. Thereby, $87.4 \%$ of the CF patients we examined live in the European part of Russia. More than $85 \%$ are Russian or come from marriages between Russians and persons belonging to other ethnic groups. According to the Russian Registry of cystic fibrosis patients of 2017 (RF CF Registry), among at least 212 pathogenic variants of the CFTR gene eleven variants are the most frequent ones in the Russian Federation (their relative frequencies exceed $1 \%$ in the sample of tested patients) and they are F508del with a share of $52.81 \%$, CFTRdele2,3-6.21\%, E92K-3.00\%, 2143delT-2.15\%, 3849+10kbC > T-2.02\%, W1282X-1.90\%, 2184insA-1.85\%, 1677delTA-1.81\%, N1303K-1.54\%, G542X-1.35\%, and L138ins with $1.24 \%$ [7]. All other CFTR variants identified in Russian patients share $12.35 \%$. The frequencies and spectrum of variants of the CFTR gene vary in different regions. This is caused by specific ethnic background of the population, as well as by different population processes occurring on different territories inhabited by the same ethnos. Thus, in the North Caucasus Federal District (NCFD), three variants are the most frequent ones: F508del (25.0\%), 1677delTA (21.5\%), and W1282X (17.2\%) [7]. A study of CFTR gene variants' spectra in different NCFD ethnic groups revealed a high proportion of variant W1282X (88\%) for Karachays [8], and variants 1677delTA (81.5\%) and E92K (12.5\%) for Chechens [9]. The most frequent variants in the Volga Federal District (VFD) are F508del (50.5\%), E92K $(8.7 \%)$ and CFTRdele2,3 (5.0\%) [7]. A high share of E92K variant in VFD is due to the prevalence of this variant for Chuvash (55\%) [10]. The second most frequent variant for Chuvash CF patients is F508del (30\%) [9], although this value is lower than in the total sample of CF patients (according to the Registry of CF patients in the Russian Federation 2017, [7]).

Russian East Slavic ethnos is the most numerous people in the Russian Federation (RF) (more than $111,000,000$ people), which makes $77.7 \%$ of the population of the country according to census of 2010 [11]. In the European part of RF, Russians make $85 \%-90 \%$ of the population.

The aim is to describe the Russian-specific spectrum of pathogenic variants of the CFTR gene, testing of which could increase the informativeness of DNA diagnostics in regions with a predominantly Russian population, as well to establish a basis for forming a patient base for possible targeted therapy. 


\section{Materials and Methods}

Initially, CFTR genotyping of 1384 CF patients (ethnic Russians) from all-Russian sample (3457 CF patients) tested in the Laboratory of Genetic Epidemiology, Research Centre for Medical Genetics were analyzed. The diagnosis of CF was made in the Scientific-Clinical Department for Cystic Fibrosis, Research Centre for Medical Genetics or in regional CF centers according to the accepted standards [10]. Diagnosis was confirmed by analysis of clinical presentation and Gibson-Cooke sweat test, with chloride ion concentrations of $60 \mathrm{mmol} / \mathrm{L}$ or higher defining positive result. The assignment of patients' Russian ancestry was based on self- or parents' reports. The study included 154 CF Russian patients, $90 \%$ of whom came from the European part of the Russian Federation and 10\% from Siberian or Far Eastern regions, for all of them at least one mutant allele was not identified.

Patients or their parents signed an informed consent to the study. The research protocol was approved by the Ethical Committee of Research Centre for Medical Genetics (Research Centre for Medical Genetics, 115522, Moscow, Moskvorechie St., 1, Russian Federation, Protocol No.17/2006 of 02.02.2006).

Molecular diagnostics consists of three consecutive stages.

First stage included analysis of 33 frequent CFTR variants (c.54-5940_273+10250del21kb (p.Ser18Argfs*16, CFTRdele2,3), c.254G>A (p.Gly85Glu, G85E), c.262_263delTT (p.Leu88IlefsX22, 394delTT), c.274G >A (p.Glu92Lys, E92K), c.350G >A (p.Arg117His, R117H), c.413_415dupTAC (p.Leu138dup; L138ins), c.472dupA (p.Ser158LysfsX5, 604insA), c.489+1G>T $(621+1 G>T)$, c.1000C > T (p.Arg334Trp, R334W), c.1040G>C (p.Arg347Pro, R347P), c.1397C>G (p.Ser466X, Ser466X), c.1519_1521delATC (p.Ile507del, I507del), c.1521_1523delCTT (p.Phe508del, F508del), c.1545_1546delTA (p.Tyr515X, 1677delTA), c.1585-1G>A (1717-1G>A), c.1624G>T (p.Gly542X, G542X), c.1652G>A (p.Gly551Asp, G551D), c.1657C>T (p.Arg553X, R553X), c.2012delT (p.Leu671X, 2143delT), c.2051_2052delAAinsG (p.Lys684SerfsX38, 2183AA>G), c.2052_2053insA (p.Gln685ThrfsX4, 2184insA), c.2657+5G >A (2789+5A >G), c.3140-16T >A (3272-16T >A), c.3476C > T (p.Ser1159Phe, S1159F), c.3475T >C (p.Ser1159Pro; S1159P), c.3535_3536insTCAA (p.Thr1179IlefsX17, 3667ins4), c.3587C >G (p.Ser1196X, S1196X), c.3691delT (p.Ser1231ProfsX4, 3821delT), c.3718-2477C>T (3849+10kbC-T), c.3816_3817delGT (p.Ser1273LeufsX28, 3944delGT), c.3844T>C (p.Trp1282Arg, W1282R), c.3846G>A (p.Trp1282X, W1282X), c.3909C>G (p.Asn1303Lys, N1303K), representing a routine Russian Federation panel that identifies up to $85 \%$ of mutant CF alleles as described previously [12].

Second stage included analysis of CFTR gene coding sequence, exon-intron junctions and 5'-UTR sequence by Sanger sequencing as described previously [12]. Variant pathogenicity status (only pathogenic or likely pathogenic variants were reported) was established using consensus recommendations of the American College of Medical Genetics and Genomics and the Association for Molecular Pathology for interpretation of sequence variants and Russian recommendations. The frequencies of identified alleles in general populations were established based on the GnomAD browser (https://gnomad.broadinstitute.org/). The predicted functional effect of missense variants was determined through SIFT, FATHMM and Radial SVM prediction algorithms as well as GERP++ and PhyloP conservation scores. Intronic and splicing variants were analyzed using Human Splicing Finder tool v. 2.4.1. Novel variants were submitted to the CFTR2 website dataset (https://cftr2.org/), CFTR1 (http://www.genet.sickkids.on.ca/cftr). Pathogenic variants of the CFTR gene are denoted according to the legacy nomenclature, besides novel variants named according to the HGVS nomenclature for NM_000492.4 (CFTR) transcript variant. 
Third stage intended to search for large rearrangements in chromosome region $7 \mathrm{q} 31.2$ (deletions/duplications-CNV) involved the CFTR gene locus by the multiplex ligation-dependent probe amplification (MLPA) method in case when no pathogenic allele was detected or an allele with uncertain significance was identified at the previous stages. MLPA analysis was performed with SALSA MLPA probemix P091-D2 CFTR (MRC-Holland, Amsterdam, the Netherlands) according to the manufacturer's recommendation. The MLPA results were analyzed using Coffalyser.Net (MRC-Holland) [12].

Variants phase was checked by segregation analysis in proband and healthy parents.

The gIVS6a_415_IVS10_987Dup26817bp (CFTRdup6b-10) duplication and its boundaries were previously described by F.M. Hantash and co-authors [13]: The fragments duplicated started $415 \mathrm{bp}$ downstream of exon 6a, in IVS6a, and spanned exons 6b, 7, 8, 9, and 10, breaking at 2987 bp downstream of exon 10 in IVS10. The duplicated region is $26,817 \mathrm{bp}$. Two pairs of primers have been developed to clarify the boundaries of CFTRdup6b-10 duplications identified in Russian CF patients. One flanks the junction area of rupture points of intron 11 (10 as in the legacy nomenclature) and intron 6a (6): IVS10F 5'-TCAGGAAATGGCAATGGGGT-3' and IVS6aR 5' -GGCTCTGGTGTGATGATCCATA-3' . A 359 bp fragment from these primers is amplified only from the allele carrying the duplication. The second pair (INT10F 5' -GGGGTTGGGAAGTGATTCCA-3' and INT10R 5'-GCCATCAGCTAGGCTTCTGTA-3') flanks the rupture area of the intron 10, amplification occurs only from the normal sequence of the intron 10 of the CFTR gene leading to a product of $234 \mathrm{bp}$.

To compare variant frequencies, the Fisher test was used. The significance level was considered to be $p \leq 0.05$.

\section{Results}

When developing a routinely used mutation panel, the laboratory's own data [14], the results of the first collaborative study [15], and studies of other Russian laboratories (in St. Petersburg [16], Bashkortostan [17], and Tomsk [18]) were considered. The panel includes 33 pathogenic variants of the CFTR gene identified in patients from different regions of the Russian Federation, as well as the variants specific for certain ethnic groups [7-10], and allows identification of up to $85 \%$ of mutant alleles in all-Russian population [12].

At the first stage, the results of testing 33 pathogenic variants of the CFTR gene in DNA of 1384 ethnic Russians with CF (previously performed in the laboratory of genetic epidemiology) were analyzed. Thereby, 29 out of 33 tested variants were revealed (Table 1). In addition to F508del and CFTRdele2,3, eight more variants can be referred to as frequent ones for ethnic Russians (frequency of variants 2143delT, 3849+10kbC-T and 2184insA exceed 2\%, variants N1303K, G542X, E92K, W1282X, and L138ins exceed 1\%). The mutation detection rate of the used panel of tested variants is $83 \%$ in the sample of ethnic Russians (Table 1). In 932 patients, two mutant variants were identified, in 426 patients only one pathogenic variant was detected, both alleles were not detected in 26 patients. 
Table 1. Frequencies of 33 variants of CFTR gene in a sample of 1384 ethnic Russians and in a nationwide sample of CF patients (RF CF Registry) [7].

\begin{tabular}{|c|c|c|c|c|}
\hline No. & Variants & Number & $\%$ & $\%$ in RF CF Registry \\
\hline 1 & c.1521_1523delCTT (p.Phe508del, F508del) & 1522 & 54.99 & 52.81 \\
\hline 2 & c.54-5940_273+10250del21kb (p.Ser18Argfs*16, CFTRdele2,3) & 210 & 7.59 & 6.21 \\
\hline 3 & c.2012delT (p.Leu671X, 2143delT) & 75 & 2.71 & 2.15 \\
\hline 4 & c. $3718-2477 \mathrm{C}>\mathrm{T}(3849+10 \mathrm{kbC}-\mathrm{T})$ & 65 & 2.35 & 2.02 \\
\hline 5 & c.2052_2053insA (p.Gln685ThrfsX4, 2184insA) & 62 & 2.24 & 1.85 \\
\hline 6 & c.3909C > G (p.Asn1303Lys, N1303K) & 48 & 1.73 & 1.54 \\
\hline 7 & c.1624G > T (p.Gly542X, G542X) & 44 & 1.59 & 1.35 \\
\hline 8 & c.274G >A (p.Glu92Lys, E92K) & 29 & 1.05 & 3.00 \\
\hline 9 & c.3846G>A (p.Trp1282X, W1282X) & 32 & 1.16 & 1.90 \\
\hline 10 & c.413_415dupTAC (p.Leu138dup; L138ins) & 31 & 1.12 & 1.24 \\
\hline 11 & c.3844T>C (p.Trp1282Arg, W1282R) & 21 & 0.76 & 0.55 \\
\hline 12 & c.1397C >G (p.Ser466X, Ser466X) & 20 & 0.72 & 0.50 \\
\hline 13 & c.3691delT (p.Ser1231Profs X4, 3821delT) & 19 & 0.69 & 0.46 \\
\hline 14 & c.1000C > T (p.Arg334Trp, R334W) & 19 & 0.69 & 0.80 \\
\hline 15 & c.262_263delTT (p.Leu88IlefsX22, 394delTT) & 15 & 0.54 & 0.94 \\
\hline 16 & c.3587C >G (p.Ser1196X, S1196X) & 14 & 0.51 & 0.48 \\
\hline 17 & c.3816_3817delGT (p.Ser1273LeufsX28, 3944delGT) & 12 & 0.43 & 0.27 \\
\hline 18 & c. $2657+5 \mathrm{G}>\mathrm{A}(2789+5 \mathrm{~A}>\mathrm{G})$ & 10 & 0.36 & 0.48 \\
\hline 19 & c. $489+1 G>T(621+1 G>T)$ & 7 & 0.25 & 0.18 \\
\hline 20 & c.3140-16T>A $(3272-16 \mathrm{~T}>\mathrm{A})$ & 6 & 0.22 & 0.34 \\
\hline 21 & c.1657C >T (p.Arg553X, R553X) & 5 & 0.18 & 0.18 \\
\hline 22 & c.1545_1546delTA (p.Tyr515X, 1677delTA) & 5 & 0.18 & 1.81 \\
\hline 23 & c.3535_3536insTCAA (p.Thr1179IlefsX17, 3667ins4) & 4 & 0.14 & 0.10 \\
\hline 24 & c.254G $>$ A (p.Gly85Glu, G85E) & 4 & 0.14 & 0.10 \\
\hline 25 & c.472dupA (p.Ser158LysfsX5, 604insA) & 3 & 0.11 & 0.10 \\
\hline 26 & c.2051_2052delAAinsG (p.Lys684SerfsX38, 2183AA>G) & 3 & 0.11 & 0.04 \\
\hline 27 & c.3475T $>$ C (p.Ser1159Pro; S1159P) & 3 & 0.11 & 0.10 \\
\hline 28 & c.1040G >C (p.Arg347Pro, R347P) & 2 & 0.07 & 0.10 \\
\hline 29 & c.350G >A (p.Arg117His, R117H) & 1 & 0.04 & 0.04 \\
\hline 30 & c.1519_1521delATC (p.Ile507del, I507del) & 0 & - & 0 \\
\hline 31 & c. $1585-1 \mathrm{G}>\mathrm{A}(1717-1 \mathrm{G}>\mathrm{A})$ & 0 & - & 0.04 \\
\hline 32 & c.1652G >A (p.Gly551Asp, G551D) & 0 & - & 0.04 \\
\hline \multirow[t]{4}{*}{33} & c.3476C > T (p.Ser1159Phe, S1159F) & 0 & - & 0.11 \\
\hline & Identified & 2290 & 82.78 & \\
\hline & Not identified & 478 & 17.22 & \\
\hline & Total & 2768 & & \\
\hline
\end{tabular}

On the second stage, 154 ethnic Russians affected by CF, for whom one or both mutant alleles were not identified when analyzing 33 mutations, were selected from the sample of 1384 CF patients for further analysis. Their genotypes were presented in Supplementary Table S2. There was a total of 164 unidentified mutant alleles of the CFTR gene.

As a result, in addition to 29 identified frequent mutations, 91 pathogenic (or likely pathogenic) genetic variants in the CFTR gene were detected (Table 2). Of these, 29 are missense mutations, 19 nonsense mutations, 14 frame-shift mutations (11 deletions and three insertions)), 17 splice-site, one in-frame insertion, 11 large rearrangements (eight deletions and three duplications). 
Table 2. The CFTR gene variants additionally identified in 154 previously screened Russian patients.

\begin{tabular}{|c|c|c|c|c|c|c|}
\hline No. & Variant According to cDNA & Protein Change & Legacy Name & Exon/Intron ${ }^{1}$ & Number & Mutation Type \\
\hline 1 & c.43delC & p.Leu15PhefsX1 & 175delC & $1 \mathrm{e}$ & 2 & sd \\
\hline 2 & c. $53+1 \mathrm{G}>\mathrm{T}$ & & $185+1 \mathrm{G}->\mathrm{T}$ & $1 \mathrm{i}$ & 2 & $\mathrm{~s}$ \\
\hline 3 & c.79G $>\mathrm{T}$ & p.Gly27X & G27X & $2 \mathrm{e}$ & 1 & $\mathrm{n}$ \\
\hline 4 & c. $115 \mathrm{C}>\mathrm{T}$ & p.Gln39X & Q39X & $2 \mathrm{e}$ & 1 & $\mathrm{n}$ \\
\hline 5 & c. $223 \mathrm{C}>\mathrm{T}$ & p.Arg75X & R75X & $3 e$ & 1 & $\mathrm{n}$ \\
\hline 6 & c. $252 \mathrm{~T}>\mathrm{A}$ & p.Tyr84X & & 3e & 2 & $\mathrm{n}$ \\
\hline 7 & c.264_268delATATT & p.Leu88PhefsX21 & & $3 e$ & 1 & sd \\
\hline 8 & c. $274-6 \mathrm{~T}>\mathrm{C}$ & & $406-6 \mathrm{~T}>\mathrm{C}$ & $3 \mathrm{i}$ & 1 & $\mathrm{~s}$ \\
\hline 9 & c. $274 \mathrm{G}>\mathrm{T}$ & p.Glu92X & E92X & $4 \mathrm{e}$ & 1 & $\mathrm{n}$ \\
\hline 10 & c. $293 \mathrm{~A}>\mathrm{G}$ & p.Gln98Arg & Q98R & $4 e$ & 1 & $\mathrm{~m}$ \\
\hline 11 & c. $358 \mathrm{G}>\mathrm{C}$ & p.Ala120Pro & & $4 \mathrm{e}$ & 1 & $\mathrm{~m}$ \\
\hline 12 & c. $422 \mathrm{C}>\mathrm{A}$ & p.Ala141Asp & A141D & $4 \mathrm{e}$ & 1 & $\mathrm{~m}$ \\
\hline 13 & c. $490-1 \mathrm{G}>\mathrm{C}$ & & & $4 \mathrm{i}$ & 1 & $\mathrm{~s}$ \\
\hline 14 & c. $580-1 \mathrm{G}>\mathrm{T}$ & & $712-1 \mathrm{G}->\mathrm{T}$ & $5 \mathrm{i}$ & 8 & $\mathrm{~s}$ \\
\hline 15 & c. $613 \mathrm{C}>\mathrm{A}$ & p.Pro205Thr & & $6 \mathrm{a} \mathrm{e}$ & 1 & $\mathrm{~m}$ \\
\hline 16 & c. $650 A>G$ & p.Glu217Gly & E217G & $6 \mathrm{a} \mathrm{e}$ & 1 & $\mathrm{~m}$ \\
\hline 17 & c. $831 \mathrm{G}>\mathrm{A}$ & p.Trp277X & & $6 \mathrm{~b} \mathrm{e}$ & 1 & $\mathrm{n}$ \\
\hline 18 & c. $940 \mathrm{G}>\mathrm{A}$ & p.Gly314Arg & G314R & $7 \mathrm{e}$ & 1 & $\mathrm{~m}$ \\
\hline 19 & c. $[1075 \mathrm{C}>\mathrm{A} ; 1079 \mathrm{C}>\mathrm{A}]$ & p.[Gln359Lys;Thr360Lys] & Q359K/T360K & $7 \mathrm{e}$ & 1 & $\mathrm{~m}$ \\
\hline 20 & c. $1083 \mathrm{G}>\mathrm{A}$ & p.Trp361X & & $7 \mathrm{e}$ & 2 & $\mathrm{n}$ \\
\hline 21 & c. $1086 \mathrm{~T}>\mathrm{A}$ & p.Tyr362X & Y362X & $7 \mathrm{e}$ & 1 & $\mathrm{n}$ \\
\hline 22 & c. $1204 \mathrm{G}>\mathrm{T}$ & p.Glu402X & & $8 \mathrm{e}$ & 1 & $\mathrm{n}$ \\
\hline 23 & c. $1209 \mathrm{G}>\mathrm{C}$ & p.Glu403Asp & E403D & $8 \mathrm{e}$ & 3 & $\mathrm{~m}$ \\
\hline 24 & c.[1210-12[5];1210-34TG[12]] & & 5T;TG12 & $7 \mathrm{i}$ & 1 & $\mathrm{~s}$ \\
\hline 25 & c.1219delG & p.Glu407AsnfsX35 & & $9 e$ & 1 & $\mathrm{sd}$ \\
\hline 26 & c. $1352 \mathrm{G}>\mathrm{T}$ & p.Gly451Val & & $9 e$ & 1 & $\mathrm{~m}$ \\
\hline 27 & c.1240_1244delCAAAA & p.Asn415X & 1365del5 & $9 \mathrm{e}$ & 11 & sd \\
\hline 28 & c. $1364 \mathrm{C}>\mathrm{A}$ & p.Ala455Glu & A455E & $9 e$ & 1 & $\mathrm{~m}$ \\
\hline 29 & c. $1382 \mathrm{G}>\mathrm{A}$ & p.Gly461Glu & & $9 \mathrm{e}$ & 1 & $\mathrm{~m}$ \\
\hline 30 & c. $1438 \mathrm{G}>\mathrm{T}$ & p.Gly480Cys & G480C & $10 \mathrm{e}$ & 1 & $\mathrm{~m}$ \\
\hline 31 & c. $1501 \mathrm{~A}>\mathrm{G}$ & p.Thr501Ala & T501A & $10 \mathrm{e}$ & 1 & $\mathrm{~m}$ \\
\hline 32 & c. $1513 \mathrm{~A}>\mathrm{C}$ & p.Asn505His & & $10 \mathrm{e}$ & 1 & $\mathrm{~m}$ \\
\hline 33 & c. $1525 \mathrm{G}>\mathrm{C}$ & p.Gly509Arg & & $10 \mathrm{e}$ & 1 & $\mathrm{~m}$ \\
\hline
\end{tabular}


Table 2. Cont.

\begin{tabular}{|c|c|c|c|c|c|c|}
\hline No. & Variant According to cDNA & Protein Change & Legacy Name & Exon/Intron ${ }^{1}$ & Number & Mutation Type \\
\hline 34 & c.1528delG & p.Val510PhefsX17 & 1660delG & $10 \mathrm{e}$ & 1 & sd \\
\hline 35 & c. $1584+1 \mathrm{G}>\mathrm{A}$ & & $1716+1 G>A$ & $10 \mathrm{i}$ & 1 & $\mathrm{~s}$ \\
\hline 36 & c. $1589 \mathrm{~T}>\mathrm{C}$ & p.Ile530Thr & & $11 \mathrm{e}$ & 1 & $\mathrm{~m}$ \\
\hline 37 & c.1608delA & p.Asp537ThrfsX3 & & $11 \mathrm{e}$ & 2 & $\mathrm{sd}$ \\
\hline 38 & c. $1646 \mathrm{G}>\mathrm{A}$ & p.Ser549Asn & $\mathrm{S} 549 \mathrm{~N}$ & $11 \mathrm{e}$ & 1 & $\mathrm{~m}$ \\
\hline 39 & c. $1705 \mathrm{~T}>\mathrm{C}$ & p.Tyr569His & $\mathrm{Y} 569 \mathrm{H}$ & $12 \mathrm{e}$ & 1 & $\mathrm{~m}$ \\
\hline 40 & c. $1735 \mathrm{G}>\mathrm{T}$ & p.Asp579Tyr & D579Y & $12 \mathrm{e}$ & 2 & $\mathrm{~m}$ \\
\hline 41 & c. $1766+2 \mathrm{~T}>\mathrm{C}$ & & & $12 \mathrm{i}$ & 2 & $\mathrm{~s}$ \\
\hline 42 & c. $1766+1 \mathrm{G}>\mathrm{A}$ & & $1898+1 G>A$ & $12 \mathrm{i}$ & 4 & $\mathrm{~s}$ \\
\hline 43 & c. $1766+1 \mathrm{G}>\mathrm{C}$ & & $1898+1 G>C$ & $12 \mathrm{i}$ & 5 & $\mathrm{~s}$ \\
\hline 44 & c.1792_1793insAAA & p.Lys598dup & K598ins & $13 \mathrm{e}$ & 1 & $\mathrm{i}$ \\
\hline 45 & c. 1795 dupA & p.Thr599AsnfsX2 & & $13 \mathrm{e}$ & 1 & $\mathrm{si}$ \\
\hline 46 & c.1911delG & p.Gln637HisfsX26 & 2043delG & $13 \mathrm{e}$ & 2 & sd \\
\hline 47 & c. $2128 \mathrm{~A}>\mathrm{T}$ & p.Lys710X & K710X & $13 \mathrm{e}$ & 3 & $\mathrm{n}$ \\
\hline 48 & c. $2195 \mathrm{~T}>\mathrm{G}$ & p.Leu732X & L732X & $13 \mathrm{e}$ & 1 & $\mathrm{n}$ \\
\hline 49 & c. $2290 \mathrm{C}>\mathrm{T}$ & p.Arg764X & $\mathrm{R} 764 \mathrm{X}$ & $13 e$ & 1 & $\mathrm{n}$ \\
\hline 50 & c. $2312 \mathrm{delA}$ & p.Asn771ThrfsX2 & & $13 \mathrm{e}$ & 1 & sd \\
\hline 51 & c. $2353 \mathrm{C}>\mathrm{T}$ & p.Arg785X & $\mathrm{R} 785 \mathrm{X}$ & $13 \mathrm{e}$ & 6 & $\mathrm{n}$ \\
\hline 52 & c. $2374 \mathrm{C}>\mathrm{T}$ & p.Arg792X & $\mathrm{R} 792 \mathrm{X}$ & $13 \mathrm{e}$ & 1 & $\mathrm{n}$ \\
\hline 53 & c. $2417 \mathrm{~A}>\mathrm{G}$ & p.Asp806Gly & D806G & $13 e$ & 1 & $\mathrm{~m}$ \\
\hline 54 & c.2589_2599delAATTTGGTGCT & p.Ile864SerfsX28 & 2721del11 & $14 \mathrm{a} \mathrm{e}$ & 2 & sd \\
\hline 55 & c. $2617 \mathrm{G}>\mathrm{T}$ & p.Glu873X & & $14 \mathrm{a} \mathrm{e}$ & 1 & $\mathrm{n}$ \\
\hline 56 & c. $2658-2 A>G$ & & $2790-2 A->G$ & $14 \mathrm{~b} \mathrm{i}$ & 1 & $\mathrm{~s}$ \\
\hline 57 & c. $2780 \mathrm{~T}>\mathrm{C}$ & p.Leu927Pro & L927P & $15 \mathrm{e}$ & 1 & $\mathrm{~m}$ \\
\hline 58 & c. $2834 \mathrm{C}>\mathrm{T}$ & p.Ser945Leu & S945L & $15 \mathrm{e}$ & 4 & $\mathrm{~m}$ \\
\hline 59 & c. $2909 \mathrm{G}>\mathrm{A}$ & p.Gly970Asp & G970D & $16 \mathrm{e}$ & 1 & $\mathrm{~m}$ \\
\hline 60 & c. $2936 \mathrm{~A}>\mathrm{T}$ & p.Asp979Val & D979V & $16 \mathrm{e}$ & 1 & $\mathrm{~m}$ \\
\hline 61 & c. $2988+1 G>A$ & & $3120+1 \mathrm{G}->\mathrm{A}$ & $16 \mathrm{i}$ & 1 & $\mathrm{~s}$ \\
\hline 62 & c. $2989-2 \mathrm{~A}>\mathrm{C}$ & & & $16 \mathrm{i}$ & 1 & $\mathrm{~s}$ \\
\hline 63 & c. $2989-2 A>G$ & & $3121-2 A->G$ & $16 \mathrm{i}$ & 1 & $\mathrm{~s}$ \\
\hline 64 & c. $3107 \mathrm{C}>\mathrm{A}$ & p.Thr1036Asn & & $17 \mathrm{a} \mathrm{e}$ & 1 & $\mathrm{~m}$ \\
\hline 65 & c. $3112 \mathrm{C}>\mathrm{T}$ & p.Gln1038X & & $17 \mathrm{a} \mathrm{e}$ & 1 & $\mathrm{n}$ \\
\hline 66 & c.3189delG & p.Trp1063X & & $17 \mathrm{~b} \mathrm{e}$ & 1 & $\mathrm{n}$ \\
\hline
\end{tabular}


Table 2. Cont.

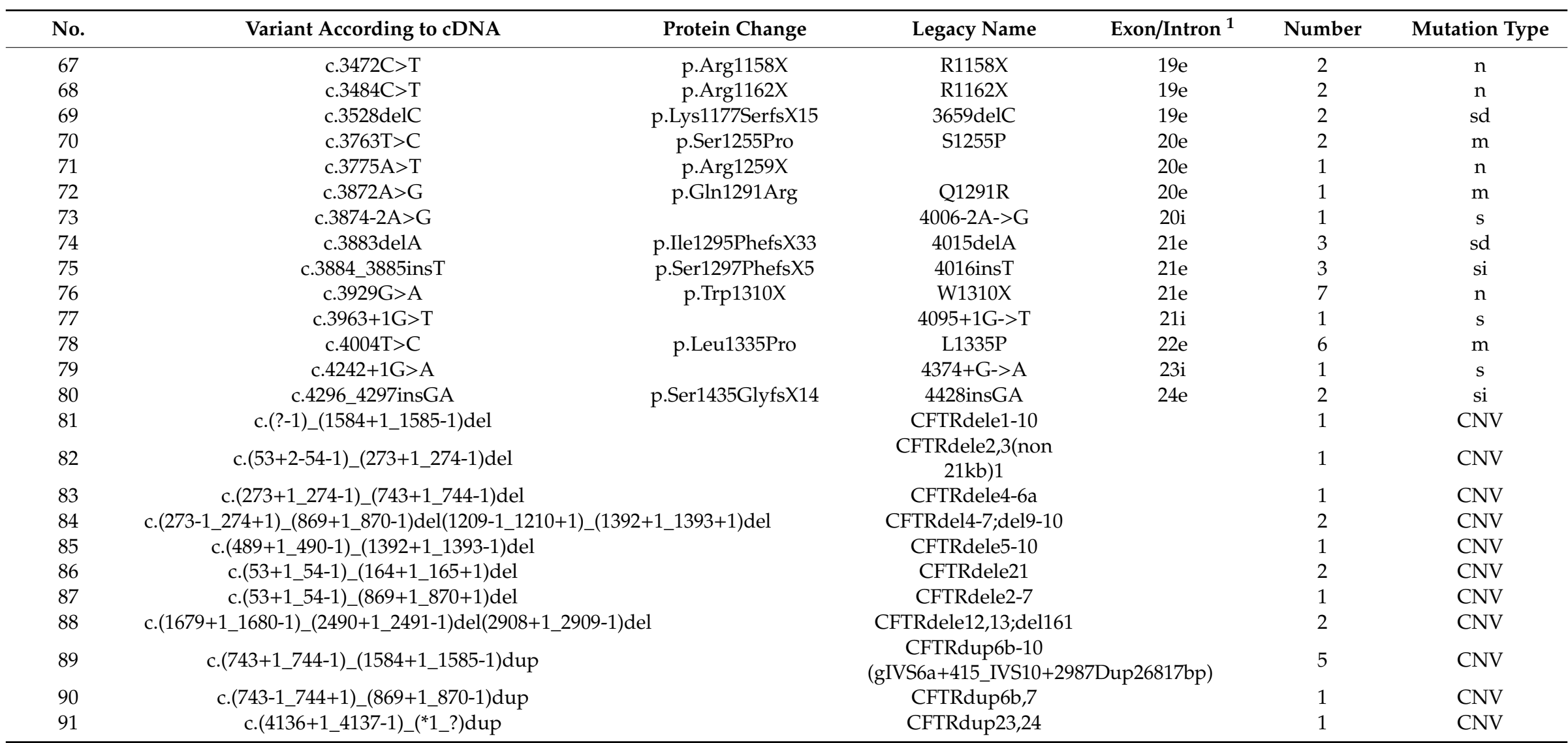

Note: $\left({ }^{1}\right)$-exon numbering according to legacy nomenclature, $(\mathrm{m})$-missense mutation, (n)-nonsense mutation, (sd)-frame-shift deletion, (si)-frame-shift insertion, (s)-splice-site, (i)-in-frame insertion, (CNV)-copy number variation (large rearrangement). 


\section{Discussion}

At present, routine DNA testing of patients includes analysis for 33 pathogenic variants in the CFTR gene (Materials and methods section). The spectrum of variants included in the first stage of molecular genetic research was developed gradually. Therefore, 1384 ethnic Russian CF patients are included in the present study, for whom all 33 variants have been tested.

The choice of the spectrum of mutations for routine analysis is conditioned by the results obtained in the course of our own studies [14], studies conducted in different laboratories of the Russian Federation on independent samples of CF patients $[6,12,16,17]$, and data on the prevalence of CFTR gene mutations in a global sample of CF patients published by the World Health Organization [18] and presented in the CFTR mutation database CFTR1 [19].

Mutations c.1521_1523delCTT (F508del), c.1624G>T (G542X), c.1652G>A (G551D), c.1657C > T (R553X), c.3846G >A (W1282X), c.3909C >G (N1303K), c.489+1G>T (621+1G>T), c.350G >A (R117H), and c.1585-1G>A (1717-1G>A), are among ones the most common in the world [18,20]. Therefore, first of all these mutations were included in the analysis of Russian patients. Variants c.1519_1521delATC (I507del), c.254G >A (G85E), c.3718-2477C > T (3849+10kbC-T), c.1000C > T (R334W), and c.1040G >C (R347P), although not among the most common in the world, are quite common for many populations with specific ethnic background. In 1993-1995, in order to detect pathogenic variants specific to the Russian population, a joint study of the coding sequence of the CFTR gene was carried out with the Institute of Biogenetics (Brest, France) by denaturing gradient gel electrophoresis with subsequent sequencing in a sample of 50 patients. It was shown that, in addition to the previously detected CFTR gene mutations, the mutations c.2012delT (2143delT), c.2052_2053insA (2184insA), c.262_263delTT (394delTT), and c.3691delT (3821delT) can also be considered frequent for ethnic Russian CF patients. [15]. In the collaborative study of Dörk T. with co-authors [21], in which our laboratory also participated, the predominant distribution of mutation c.54-5940_273+10250del21kb (CFTRdele2,3) was shown for the populations of Eastern Europe and the relative frequency of this mutation was determined for the studied Russian patients (7.2\%); second in frequency after the mutation c.1521_1523delCTT (F508del). T.E. Ivashchenko [16] for the first time describes the variants c.1545_1546delTA (1677delTA), c.3587C >G (S1196X) and c.3844T>C (W1282R), relatively frequent for CF patients from Russia. The variant c.1545_1546delTA (1677delTA) was shown to be common for Georgian patients, whereas variants c.3587C >G (S1196X) and c.3844T >C (W1282R) were identified for Russian CF patients. In a study conducted in our laboratory, variants c.3535_3536insTCAA (3667ins4), c.3816_3817delGT (3944delGT), c.472dupA (604insA), and c.413_415dupTAC (L138ins) were identified and included in the frequent mutations' panel [14].

\subsection{Similarity and Difference of Frequency Profiles of Common CF Variants in Two Samples of Russian Patients and the Data of CFTR2}

A comparison of frequency profiles of 33 variants tested at the first stage shows similarity of frequency distributions for ethnic Russian patients and for patients of All-Russian sample (Table 1): the most frequent is c.1521_1523delCTT (F508del) (54.99\% and 52.81\%, respectively), the second in frequency is c.54-5940_273+10250del21kb (CFTRdele2,3) (7.59\% and 6.21\%), and frequencies of eight more variants exceed 1\%. This similarity is not surprising, as ethnic Russians make up the majority (over 85\%) of CF patients in the Russian Federation. However, there also are differences. Frequencies of the variants c.1521_1523delCTT (F508del) $(p=0.059)$, c.54-5940_273+10250del21kb (CFTRdele2,3) $(p=0.018)$, c.2012delT (2143delT) $(p=0.109)$, c.3718-2477C $>$ T $(3849+10 \mathrm{kbC}>\mathrm{T}), \mathrm{c} .2052 \_2053$ insA (2184insA), c.3909C >G (N1303K), c.1624G > T (G542X), c.3844T>C (W1282R), c.1397C>G (pSer466X), c.3691delT (3821delT), c.3816_3817delGT (3944delGT) are higher for ethnic Russian patients' sample than for the all-Russian one (Table 1, Figure 1). Perhaps, this is due to the fact that these variants are typical for ethnic Russians and may reflect this ancestry. While frequencies of other variants prevail in the all-Russian sample, which reflects the fact that these variants prevail among patients belonging to other ethnic groups. Thus, the frequency of variant c.1545_1546delTA (1677delTA) 
for ethnic Russians is much lower than for the all-Russian sample $(0.18 \%$ and $1.81 \%$, respectively, $p<0.0001)$. The variant c.1545_1546delTA (1677delTA) is predominantly distributed in the North Caucasus populations (Chechens, Ingush, Kumyks) [9,12]. The frequency of variant c.262_263delTT (394delTT) for ethnic Russians is lower than for the all-Russian sample $(0.54 \%$ vs. $0.94 \%, p=0.074$, although difference is not significant). In the Russian Federation, it is more often found among the population associated with the past settlement of the Finno-Ugric peoples in northwestern European regions and in the Volga-Ural region [12,17]. The frequency of variant c.274G $>$ A (E92K) for ethnic

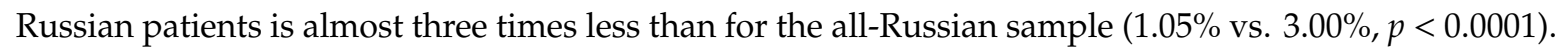
The frequency of variant c.274G>A (p.Glu92Lys, E92K) is maximum for Chuvash (up to 55\%) [10], high for Tatars (6.67\%), Bashkirs (6.25\%) [7], Chechens (12.5\%) [9]. The frequency of c.3846G>A (p.Trp1282X, W1282X) is significant higher in the all-Russian sample (RF CF Registry) than in ethnic Russian patients $(1.16 \%$ vs. $1.90 \%, p=0.012)$.

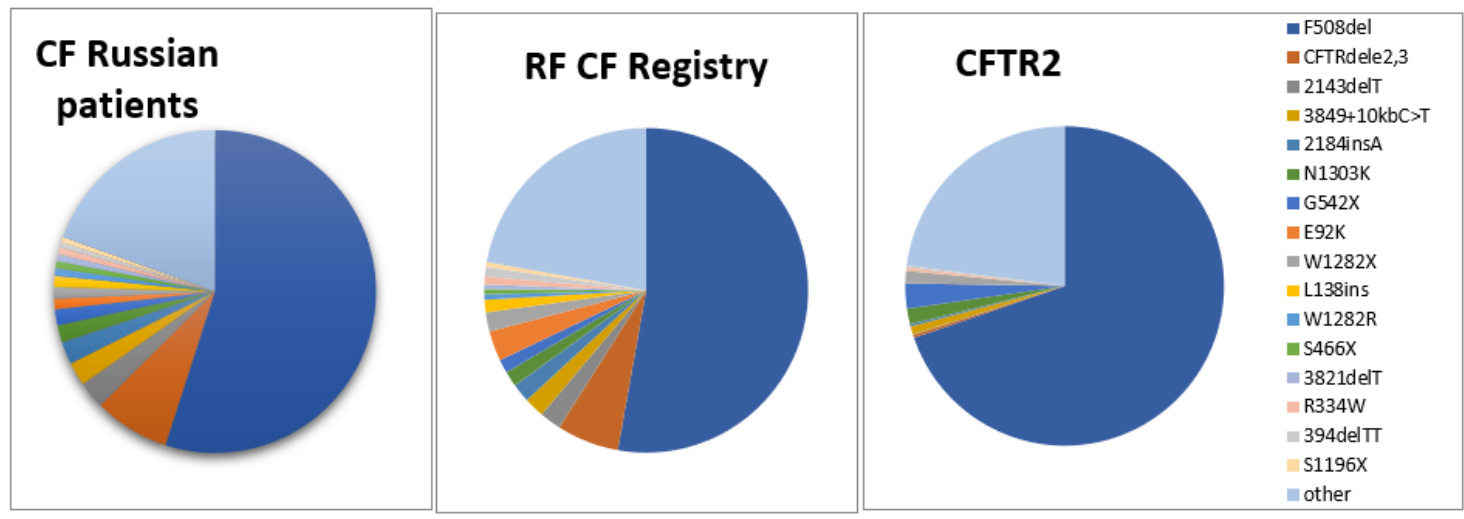

Figure 1. Frequency distribution of CFTR gene variants in three samples of CF patients: ethnic Russians, CF RF Registry, CFTR2 database [22].

When comparing CF-causing variant frequencies in ethnic Russian CF patients to the CFTR2 database [22], significant frequency difference was found (Fig. 1, Supplementary Table S1). So, the frequencies of c.54-5940_273+10250del21kb (p.Ser18Argfs*16, CFTRdele2,3), c.2012delT (p.Leu671X, 2143delT), c.3718-2477C>T (3849+10kbC-T), c.2052_2053insA (p.Gln685ThrfsX4, 2184insA), c.274G >A (p.Glu92Lys, E92K), c.413_415dupTAC (p.Leu138dup; L138ins) and some other variants appear higher in ethnic Russian patients while the frequencies of c.1521_1523delCTT (p.Phe508del, F508del), c.1624G>T (p.Gly542X, G542X), c.2657+5G>A (2789+5A>G), c.489+1G>T (621+1G>T), c.1657C>T (p.Arg553X, R553X), c.254G>A (p.Gly85Glu, G85E), c.1040G>C (p.Arg347Pro, R347P), c.350G>A (p.Arg117His, $\mathrm{R} 117 \mathrm{H})$ were lower. Variants c.3844T $>\mathrm{C}$ (p.Trp1282Arg, W1282R), c.3140-16T $>A$ (3272-16T $>A)$, and c.3816_3817delGT (p.Ser1273LeufsX28, 3944delGT) were not listed in CFTR2. Variants c.1652G $>$ A (p.Gly551Asp, G551D), c.1585-1G>A (1717-1G>A), and c.3476C>T (p.Ser1159Phe, S1159F) were not found in tested cohort of Russian patients (Supplementary Table S1). However, the differences in frequencies in these latter series involve rare variants and their significance remains unknown.

\subsection{Sanger Sequencing Detection of the CFTR Gene Variants}

As a result of analysis of the coding sequence and regions of exon-intron junctions 80 variants in addition to preliminary tested common CFTR gene variants were identified. 61 variants identified in this work were identified on one chromosome and 17 on two chromosomes (Table 2). Each of the variants c.1209G >C (E403D), c.2128A > T (K710X), c.3883delA (4015delA) and c.3884_3885insT (4016insT) were detected for three, c.1766+1G >A $(1898+1 \mathrm{G}>\mathrm{A})$ and c.2834C $>$ T (S945L) for four, c.1766+1G $>C$ (1898+1G>C) and c.(743+1_744-1)_(1584+1_1585-1)dup (CFTRdup6b-10) for five, c.2353C >T (R785X) and c.4004T >C (L1335P) for six, c.3929G >A (W1310X) for seven, c.580-1G > T (712-1G > T) for eight, and c.1240_1244delCAAAA (1365del5) for 11 unrelated patients (Table 2). 
Some of genetic variants identified in sequencing were first discovered in this study. Description of 15 is presented in a previously published paper [23]. Nine of these variants are nonsense mutations (c.252T>A (p.Tyr84X), c.831G>A (p.Trp277X), c.1083G >A (p.Trp361X), c.3112C>T (p.Gln1038X)) or frame-shift mutations (c.264_268delATATT (p.Leu88PhefsX21), c.1219delG (p.Glu407AsnfsX35), c.1608delA (p.Asp537ThrfsX3), c.1795dupA (p.Thr599AsnfsX2), c.3189delG (p.Trp1063X), resulting in the formation of premature stop codon (Table 1). Variant c.490-1G $>$ C breaks the acceptor site of 5 exon splicing. These variants belong to the category of PVS1 null variants (pathogenic variant sequence) according to the criteria of classification of pathogenicity of genetic variants [24]. Variant c.1792_1793insAAA (p.Lys598dup) leads to the insertion of lysine into position 598, and clinical significance of the variant is assessed as pathogenic. The clinical significance of the missense mutations (c.358G >C (p.Ala120Pro), c.1382G >A (p.Gly461Glu), c.1513A >C (p.Asn505His), c.1525G>C (p.Gly509Arg)) is assessed as probably pathogenic.

Eight more variants are presented for the first time. Two variants-nonsense mutations (c.1204G $>\mathrm{T}$ (p.Glu402X), c.2617G>T (p.Glu873X)) and one deletion with frame shift (c.2312delA (p.Asn771ThrfsX2)) are concluded to be PVS1 null variants according to the ACMG classification. Variant c.2989-2A $>C$ is a violation of the 19 exon splicing site. Three missense mutations (c.613C $>A$ (p.Pro205Thr), c.1352G $>T$ (p.Gly451Val), c.1589T>C (p.Ile530Thr), c.3107C >A (p.Thr1036Asn)), the clinical significance of which is assessed as probably pathogenic according to the recommendations [24]. The characteristics of the phenotypes of patients who carry rare missense variants are presented in Supplementary Table S3.

\subsection{CNV in Russian CF Patients Detected by MLPA}

Large rearrangements of the CFTR gene were found for 18 unrelated patients, which is $10.8 \%$ (18/166) of the tested mutant alleles and should account for about $1 \%$ in the total sample of all mutant alleles in Russians. The MLPA method revealed 11 large rearrangements of the CFTR gene: three duplications and eight deletions (Table 1). Four of the large rearrangements were detected in several families. Thus, the duplication of a fragment covering 7-11 (6b-10) exons was detected for five unrelated patients. The testing system we developed allowed us to confirm that the duplication detected had the same frames as previously described in the literature [13]. In the RF CF Registry 2017, this variant was noted for six more unrelated patients. Thus, CFTRdup6b-10 was detected in eleven unrelated patients. Six of them live in the Volga-Ural region, three in the Central region. It should be noted that two patients from the Volga-Ural region belong to the other ethnic groups: one-Bashkir and one-Udmurt.

Each of the deletions, c.(53+1_54-1)_(164+1_165-1)del (CFTRdele2), c.[(1679-1_1680+1)_(2490+1 _2491-1)del[;](2908+1_2989-1)del] (CFTRdele12,13;del16) and c.(273-1_274+1)_(869+1_870-1)del(1209-1 _1210+1)_(1392+1_1393+1)del (CFTRdel4-7;del9-10) was detected twice. Complex deletion, CFTRdele12,13;del16, was detected for two patients from unrelated families living in the Moscow region; deletion CFTRdel4-7; de19-10 for two families from the Kaliningrad region and the Republic of Buryatia; deletion CFTRdele2 in families from the Transbaikal region and Irkutsk region.

\subsection{Detection Rate of Three-Stage Analysis of CFTR Gene in Russian CF Patients}

As a result of analysis of the coding sequence and regions of exon-intron junctions and subsequent search for large rearrangements, 163 out of 164 alleles were identified that were not detected after preliminary testing of frequent variants of the CFTR gene.

In one patient only variant E217G with the F508del in trans was detected after sequencing and MLPA. In NCBI-ClinVar database variant E217G is considered to be variant of conflicting interpretation of pathogenicity (benign; likely benign; uncertain significance) [25]. In the study by Lee J.H. et al. [26] it was shown that non-synonymous E217G mutation in the M470 background caused a $60 \%-80 \%$ reduction in CFTR-dependent $\mathrm{Cl}^{-}$currents and $\mathrm{HCO}_{3}{ }^{-}$transport activities. So we might suggest that the clinical presentation in that patient is due to complex allele E217G-M470 (Supplementary Table S3). 
The second mutant allele of the CFTR gene could not be identified in one sample. Failure to identify the second pathologic mutation in the CFTR gene after sequencing the coding sequence and searching for large rearrangements may be due to the location of the pathogenic variant either in inner regions of the introns, or in regulatory regions of the CFTR gene, or in regulatory regions outside the CFTR gene. Indeed, such variants have been recently identified, for example, c.1680-883A>G, c.2989-313A $>$ T, c.3469-1304C $>$ G, or c.3874-4522A $>$ G, that lead to the creation of a new donor splice site and the activation of a cryptic acceptor splice site, resulting in the inclusion of an additional pseudo-exon (PE) and the loss of wild type (WT) CFTR transcripts [27].

\section{Conclusions}

In a representative sample of CF patients (ethnic Russians), the spectrum of 33 routinely analyzed (in Russia) variants of the CFTR gene was studied. It was shown that, out of 29 identified variants, frequencies of only 10 exceed $1 \%$, and the mutation detection rate of testing did not exceed $85 \%$. Consistent use of sequencing and MLPA methods has allowed us to identify a significant variety of CFTR gene mutations spectrum (91 additional genetic variants), to expand the spectrum of frequent variants $(\mathrm{c} .1766+1 \mathrm{G}>\mathrm{C}(1898+1 \mathrm{G}>\mathrm{C}), \mathrm{c} .2353 \mathrm{C}>\mathrm{T}$ (R785X), c.(743+1_744-1)_(1584+1_1585-1)dup (CFTRdup6b-10), c.4004T>C (L1335P), c.3929G >A (W1310X), c.580-1G $>$ T (712-1G $>$ T), c.1240_1244delCAAAA (1365del5), detected for five and more unrelated patients, to increase the detection rate of identified mutant alleles for Russian CF patients up to $99.4 \%$, consistently using the strategy of Sanger sequencing and MLPA analysis. This information can be useful for the further optimization of medical genetic counseling in CF high-risk families, for improving the neonatal screening program for $\mathrm{CF}$, and for making decision about the possible $\mathrm{CFTR}$ modulators therapy in the future. The identification of previously unknown CF-pathogenic or likely-pathogenic variants is a useful piece of information for diagnostic testing not only in Russia, but worldwide, and can be considered as a contribution to the general knowledge about the CFTR variant heterogeneity.

Supplementary Materials: The following are available online at http://www.mdpi.com/2073-4425/11/5/554/s1, Table S1: Frequencies of 33 variants of CFTR gene in the samples of 1384 ethnic Russians and in the CF patients from CFTR2 database [22], Table S2: Genotypes and CFTR gene variants in 154 Russian patients tested for 33 common CFTR variants, Table S3: Clinical and demographic characteristics of CF patients with rare missense variants.

Author Contributions: Data curation, N.V.P. and N.Y.K.; Formal analysis, N.V.P. and T.A.V.; Funding acquisition, S.I.K. and R.A.Z.; Investigation, N.V.P.; Project administration, E.K.G., S.I.K. and R.A.Z.; Resources, N.Y.K., E.I.K., E.K.Z., A.Y.V., V.D.S. and V.A.G.; Supervision, E.K.G. and R.A.Z.; Validation, T.A.V. and A.V.M.; Writing-original draft, N.V.P.; Writing-review \& editing, A.V.M. All authors have read and agreed to the published version of the manuscript.

Funding: The research was partially supported by grant RFBR 20-015-0061A and within the state task of the Ministry of education and science of Russia.

Acknowledgments: The authors are grateful to Stanislav Krasovsky and Elena Amelina for their input to the collection of the clinical data of adult CF patients. Also the authors would like to thank the National CF Patient Registry for providing access to patients data and thank the individual regional CF centers representatives for allowing the use of data, https://mukoviscidoz.org/mukovistsidoz-vrossii.html.

Conflicts of Interest: The authors declare no conflict of interest. The funders had no role in the design of the study; in the collection, analyses, or interpretation of data; in the writing of the manuscript, or in the decision to publish the results.

\section{References}

1. Sosnay, P.R.; Raraigh, K.S.; Gibson, R.L. Molecular Genetics of Cystic Fibrosis Transmembrane Conductance Regulator: Genotype and Phenotype. Pediatr. Clin. North Am. 2016, 63, 585-598. [CrossRef] [PubMed]

2. Farrell, P.M.; White, T.B.; Howenstine, M.S.; Munck, A.; Parad, R.B.; Rosenfeld, M.; Sommerburg, O.; Accurso, F.J.; Davies, J.C.; Rock, M.J.; et al. Diagnosis of Cystic Fibrosis in Screened Populations. J. Pediatr. 2017, 181S, S33-S44. [CrossRef] [PubMed] 
3. Castellani, C.; Cuppens, H.; Macek, M., Jr.; Cassiman, J.J.; Kerem, E.; Durie, P.; Tullis, E.; Assael, B.M.; Bombieri, C.; Brown, A.; et al. Consensus on the use and interpretation of cystic fibrosis mutation analysis in clinical practice. J. Cyst. Fibros. 2008, 7, 179-196. [CrossRef] [PubMed]

4. De Boeck, K.; Vermeulen, F.; Dupont, L. The diagnosis of cystic fibrosis. Presse Med. 2017, 46, e97-e108. [CrossRef] [PubMed]

5. Castellani, C.; Duff, A.J.A.; Bell, S.C.; Heijerman, H.G.M.; Munck, A.; Ratjen, F.; Sermet-Gaudelus, I.; Southern, K.W.; Barben, J.; Flume, P.A.; et al. ECFS best practice guidelines: The 2018 revision. J. Cyst. Fibros. 2018, 17, 153-178. [CrossRef] [PubMed]

6. National Consensus Project. Cystic Fibrosis: Definition, Diagnostic Criteria, Treatment 2018; Kondratyeva, E.I., Kashirskaya, N.Y., Kaprqanov, N.I., Eds.; Company BORGES Ldt.: Moscow, Russia, 2018; p. 356.

7. MEDPRAKTIKA-M. Registry of Patients with Cystic Fibrosis in the Russian Federation. 2017; Voronkova, A.Y., Amelina, E.A., Kashirskaya, N.Y., Kondratyeva, E.I., Krasovsky, S.A., Starinova, M.A., Kapranov, N.I., Eds.; MEDPRAKTIKA-M: Moscow, Russia, 2019; p. 68. (in Russian)

8. Petrova, N.V.; Kashirskaya, N.Y.; Vasilieva, T.A.; Timkovskaya, E.E.; Voronkova, A.Y.; Shabalova, L.A.; Kondrateva, E.I.; Sherman, V.D.; Kapranov, N.I.; Zinchenko, R.A.; et al. High proportion of W1282X mutation in CF patients from Karachai-Cherkessia. J. Cyst. Fibros. 2016, 15, e28-e32. [CrossRef] [PubMed]

9. Petrova, N.V.; Kashirskaya, N.Y.; Saydaeva, D.K.; Polyakov, A.V.; Adyan, T.A.; Simonova, O.I.; Gorinova, Y.V.; Kondratyeva, E.I.; Sherman, V.D.; Novoselova, O.G.; et al. Spectrum of CFTR mutations in Chechen cystic fibrosis patients: High frequency of c.1545_1546delTA (p.Tyr515X; 1677delTA) and c.274G>A (p.Glu92Lys, E92K) mutations in North Caucasus. BMC Med. Genet. 2019, 20, 44. [CrossRef]

10. Stepanova, A.A.; Abrukova, A.V.; Savaskina, E.N.; Poliakov, A.V. Mutation p.E92K is the primary cause of cystic fibrosis in Chuvashes. Russ. J. Genet. 2012, 48, 731-737. [CrossRef]

11. StatData.ru. Available online: http://www.statdata.ru/nacionalnyj-sostav-rossii (accessed on 8 February 2020).

12. Petrova, N.V.; Kondratyeva, E.I.; Krasovsky, S.A.; Polyakov, A.V.; Ivachshenko, T.E.; Pavlov, A.E.; Zinchenko, R.A.; Ginter, E.K.; Kutsev, S.I.; Odinokova, O.N.; et al. National Consensus Project «Cystic fibrosis: Definition, diagnostic criteria, treatment» Section «Genetics of Cystic Fibrosis. Molecular genetic diagnosis of cystic fibrosis». Meditsinskaya Genet. 2016, 11, 29-45. (in Russian).

13. Hantash, F.M.; Redman, J.B.; Goos, D.; Kammesheidt, A.; McGinniss, M.J.; Sun, W.; Strom, C.M. Characterization of a recurrent novel large duplication in the cystic fibrosis transmembrane conductance regulator gene. J. Mol. Diagn. 2007, 9, 556-560. [CrossRef]

14. Petrova, N.V.; Timkovskaya, E.E.; Zinchenko, R.A. The analysis of CFTR mutations frequencies in different populations of Russia. Eur. J. Hum. Genet. 2008, 16, 387.

15. Verlingue, C.; Kapranov, N.I.; Mercier, B.; Ginter, E.K.; Petrova, N.V.; Audrezet, M.P.; Ferec, C. Complete screening of mutations in the coding sequence of the CFTR gene in a sample of CF patients from Russia: Identification of three novel alleles. Hum. Mutat. 1995, 5, 205-209. [CrossRef] [PubMed]

16. Ivaschenko, T.E.; Baranov, V.S. Biochemical and Molecular Genetic Basis of Cystic Fibrosis Pathogenesis; Intermedika: Saint-Petersburg, Russia, 2002; p. 256. (in Russian)

17. Korytina, G.F.; Victorova, T.V.; Baykova, G.V.; Khusnutdinova, E.K. Analysis of the Spectra of Mutations and Polymorphic Loci of Cystic Fibrosis Transmembrane Conductance Regulator in the Population of Bashkortostan. Rus. J. Genet. 2002, 38, 1076-1081. [CrossRef]

18. WHO Human Genetics Programme. The Molecular Genetic Epidemiology of Cystic Fibrosis. Report of a Joint Meeting of WHO/ECFTN/ICF(M)/ECFS/World Health Organization (WHO); WHO: Genoa, Italy, 2002; Available online: https://www.cfww.org/docs/who/2002/who_hgn_cf_wg_04.02.pdf (accessed on 8 February 2020).

19. Cystic Fibrosis Mutation Database. Available online: http://www.genet.sickkids.on.ca (accessed on 5 February 2020).

20. Bobadilla, J.L.; Macek, M., Jr.; Fine, J.P.; Farrell, P.M. Cystic fibrosis: A worldwide analysis of CFTR mutations-Correlation with incidence data and application to screening. Hum. Mutat. 2002, 19, 575-606. [CrossRef] [PubMed]

21. Dörk, T.; Macek, M., Jr.; Mekus, F.; Tümmler, B.; Tzountzouris, J.; Casals, T.; Krebsová, A.; Koudová, M.; Sakmaryová, I.; Macek, M.S.; et al. Characterization of a novel 21-kb deletion, CFTRdele2,3(21kb), in the CFTR gene: A cystic fibrosis mutation of Slavic origin common in Central and East Europe. Hum. Genet. 2000, 106, 259-268. [CrossRef] [PubMed] 
22. CFTR2. Clinical and Functional Translation of CFTR. Available online: https://www.cftr2.org/ (accessed on 5 February 2020).

23. Petrova, N.V.; Marakhonov, A.V.; Vasilyeva, T.A.; Kashirskaya, N.Y.; Ginter, E.K.; Kutsev, S.I.; Zinchenko, R.A. Comprehensive genotyping reveals novel CFTR variants in cystic fibrosis patients from the Russian Federation. Clin. Genet. 2018, 95, 1-4. [CrossRef] [PubMed]

24. Richards, S.; Aziz, N.; Bale, S.; Bick, D.; Das, S.; Gastier-Foster, J.; Grody, W.W.; Hegde, M.; Lyon, E.; Spector, E.; et al. Standards and guidelines for the interpretation of sequence variants: A joint consensus recommendation of the American College of Medical Genetics and Genomics and the Association for Molecular Pathology. Genet. Med. 2015, 17, 405-424. [CrossRef] [PubMed]

25. National Center for Biotechnology Information (NCBI-ClinVar). Available online: https://www.ncbi.nlm.nih. gov/clinvar/ (accessed on 5 February 2020).

26. Lee, J.H.; Choi, J.H.; Namkung, W.; Hanrahan, J.W.; Chang, J.; Song, S.Y.; Park, S.W.; Kim, D.S.; Yoon, J.-H.; Suh, Y.; et al. A haplotype-based molecular analysis of CFTR mutations associated with respiratory and pancreatic diseases. Hum. Mol. Genet. 2003, 12, 2321-2332. [CrossRef] [PubMed]

27. Bergougnoux, A.; Délétang, K.; Pommier, A.; Varilh, J.; Houriez, F.; Altieri, J.P.; Koenig, M.; Férec, C.; Claustres, M.; Lalau, G.; et al. Functional characterization and phenotypic spectrum of three recurrent disease-causing deep intronic variants of the CFTR gene. J. Cyst. Fibros. 2019, 18, 468-475. [CrossRef] [PubMed]

(C) 2020 by the authors. Licensee MDPI, Basel, Switzerland. This article is an open access article distributed under the terms and conditions of the Creative Commons Attribution (CC BY) license (http://creativecommons.org/licenses/by/4.0/). 